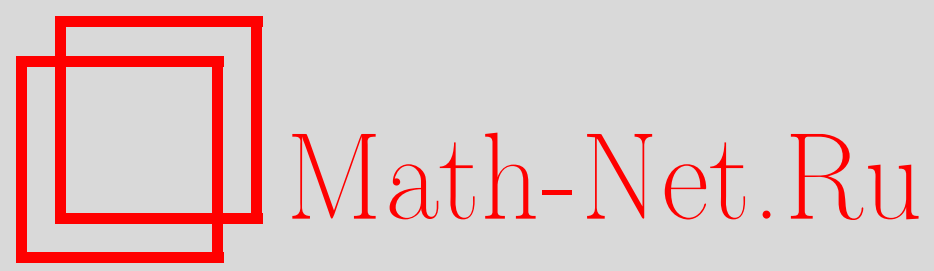

Е. А. Уткина, Об одной трёхмерной задаче Гурса, Вестн. Сам. гос. техн. унта. Сер. Физ.-мат. науки, 2001, выпуск 12, 30-35

DOI: https://doi.org/10.14498/vsgtu58

Использование Общероссийского математического портала Math-Net.Ru подразумевает, что вы прочитали и согласны с пользовательским соглашением

http://www . mathnet.ru/rus/agreement

Параметры загрузки:

IP : 3.89 .185 .249

26 апреля 2023 г., 14:07:06 


\section{Е.А. Уткина}

\section{ОБ ОДНОЙ ТРЕХМЕРНОЙ ЗАДАЧЕ ГУРСА}

Для дифференцииального уравнения шестого порядка в $E_{3}$ со старшей частной производной в случае двукратных характеристик разрабатывается вариант метода Римана, позволяющий получить решения задачи Гурса. Указываются частные случаи, когда функиия Римана (а, следовательно, и решение рассматриваемой задачи) может быть записано в явном виде.

В области $D=\left\{x_{0}<x<x_{1}, y_{0}<y<y_{1}, z_{0}<z<z_{1}\right\}$ рассмотрим уравнение с переменными коэффициентами

$$
L(u) \equiv \sum_{\alpha, \beta, \gamma=0}^{2} a_{\alpha \beta \gamma}(x, y, z) \frac{\partial^{\alpha+\beta+\gamma} u}{\partial x^{\alpha} \partial y^{\beta} \partial z^{\gamma}}=F(x, y, z) .
$$

Его можно считать пространственным обобщением уравнения, изучавшегося в [1-8]. Наиболее общие результаты получены в [7]. С другой стороны, (1) можно считать усложнением уравнения из работ [8-10] со старшей производной $u_{x y z}$, которое играет важную роль в теории аппроксимации и отображений и при описании процессов вибрации [12, с.109]. К подобному же уравнению сводится задача интегрального представления преобразований одних обыкновенных линейных дифференциальных операторов в другие [13].

1. Пусть $X, Y, Z$ - грани $D$ при $x=x_{0}, y=y_{0}, z=z_{0}$ соответственно. Будем считать, что $a_{221} \equiv 1$, а гладкость остальных коэффициентов (1) определяется включениями

$$
a_{\alpha \beta \gamma} \in C^{\alpha+\beta+\gamma}(\bar{D}), F \in C^{2+2+1}(\bar{D}) .
$$

Здесь $C^{\alpha+\beta+\gamma}$ описывает класс непрерывных в $\bar{D}$ вместе с их производными $\partial^{r+s+t} / \partial x^{r} \partial y^{s} \partial z^{t}(r=0, \ldots, \alpha ; s=0, . ., \beta ; t=0, \ldots, \gamma)$ функций.

3 а д а ч а (Г у р с а). Найти в классе $C^{2+2+2}(D)$ решение уравнения (1), удовлетворяющее граничным условиям

$$
\begin{aligned}
& \left.u\right|_{X}=\varphi(y, z),\left.u\right|_{Y}=\psi(x, z),\left.u\right|_{Z}=\theta(x, y), \\
& \left.u_{x}\right|_{X}=\varphi_{1}(y, z),\left.u_{y}\right|_{Y}=\psi_{1}(x, z),\left.u_{z}\right|_{Z}=\theta_{1}(x, y), \\
& \theta, \theta_{1} \in C^{2+2}(\bar{Z}), \varphi, \varphi_{1} \in C^{2+2}(\bar{X}), \psi, \psi_{1} \in C^{2+2}(\bar{Y}) .
\end{aligned}
$$

Полагаем на ребрах $D$ выполнение условий согласования

$$
\varphi\left(y_{0}, z\right)=\psi\left(x_{0}, z\right), \varphi\left(y, z_{0}\right)=\theta\left(x_{0}, y\right), \psi\left(x, z_{0}\right)=\theta\left(x, y_{0}\right),
$$

при этом сами согласованные значения непрерывно дифференцируемы.

Решение данной задачи будем осуществлять путем развития варианта метода Римана $[8,10]$.

Функцией Римана $R(x, y, z, \xi, \eta, \varsigma)$ назовем решение интегрального уравнения

$$
\begin{gathered}
V(x, y, z)-\int_{\varsigma}^{z}\left[a_{221}(x, y, \gamma) V(x, y, \gamma)-(z-\gamma) a_{220}(x, y, \gamma) V(x, y, \gamma)\right] d \gamma- \\
-\int_{\eta}^{y}\left[a_{212}(x, \beta, z) V(x, \beta, z)-(y-\beta) a_{202}(x, \beta, z) V(x, \beta, z)\right] d \beta-\int_{\xi}^{x}\left[a_{122}(\alpha, y, z) V(\alpha, y, z)-\right. \\
\left.-(x-\alpha) a_{022}(\alpha, y, z) V(\alpha, y, z)\right] d \alpha+\int_{\xi}^{x} \int_{\eta}^{y}\left[a_{112}(\alpha, \beta, z) V(\alpha, \beta, z)-\right. \\
-(y-\beta) a_{102}(\alpha, \beta, z) V(\alpha, \beta, z)-(x-\alpha) a_{012}(\alpha, \beta, z) V(\alpha, \beta, z)+ \\
\left.+(x-\alpha)(y-\beta) a_{002}(\alpha, \beta, z) V(\alpha, \beta, z)\right] d \beta d \alpha+
\end{gathered}
$$


$+\int_{\xi}^{x} \int_{\zeta}^{z}\left[a_{121}(\alpha, y, \gamma) V(\alpha, y, \gamma)-(z-\gamma) a_{120}(\alpha, y, \gamma) V(\alpha, y, \gamma)-\right.$ $\left.-(x-\alpha) a_{021}(\alpha, y, \gamma) V(\alpha, y, \gamma)+(x-\alpha)(z-\gamma) a_{020}(\alpha, y, \gamma) V(\alpha, y, \gamma)\right] d \gamma d \alpha+$

$+\int_{\eta}^{y} \int_{\zeta}^{z}\left[a_{211}(x, \beta, \gamma) V(x, \beta, \gamma)-(y-\beta) a_{201}(x, \beta, \gamma) V(x, \beta, \gamma)\right.$

$\left.-(z-\gamma) a_{210}(x, \beta, \gamma) V(x, \beta, \gamma)+(y-\beta)(z-\gamma) a_{200}(x, \beta, \gamma) V(x, \beta, \gamma)\right] d \gamma d \beta-$

$-\int_{\xi}^{x} \int_{\eta}^{y} \int_{\zeta}^{z}\left[a_{111}(\alpha, \beta, \gamma) V(\alpha, \beta, \gamma)-(y-\beta) a_{101}(\alpha, \beta, \gamma) V(\alpha, \beta, \gamma)-\right.$

$-(x-\alpha) a_{011}(\alpha, \beta, \gamma) V(\alpha, \beta, \gamma)-(z-\gamma) a_{110}(\alpha, \beta, \gamma) V(\alpha, \beta, \gamma)+$

$+(y-\beta)(z-\gamma) a_{100}(\alpha, \beta, \gamma) V(\alpha, \beta, \gamma)+(x-\alpha)(z-\gamma) a_{010}(\alpha, \beta, \gamma) V(\alpha, \beta, \gamma)+$ $+(x-\alpha)(y-\beta) a_{001}(\alpha, \beta, \gamma) V(\alpha, \beta, \gamma)-$

$\left.-(x-\alpha)(y-\beta)(z-\gamma) a_{000}(\alpha, \beta, \gamma) V(\alpha, \beta, \gamma)\right] d \gamma d \beta d \alpha=1$,

которое существует и единственно [14, с.180].

Непосредственной проверкой можно убедиться в выполнении тождества для любой функции $u$ из класса $C^{2+2+2}(D)$ :

$$
\begin{gathered}
(u R)_{x x y y z z} \equiv R L(u)+\left(u A_{1}\right)_{x x y y z}+\left(u A_{2}\right)_{x x y z z}+\left(u A_{3}\right)_{x y y z z}-\left(u B_{1}\right)_{x y z z}-\left(u B_{2}\right)_{x x y z}- \\
-\left(u B_{3}\right)_{x y y z}-\left(u B_{4}\right)_{x x y y}-\left(u B_{5}\right)_{x x z z}-\left(u B_{6}\right)_{y y z z}+\left(u C_{1}\right)_{x y z}+\left(u C_{2}\right)_{x z z}+ \\
+\left(u C_{3}\right)_{x y y}+\left(u C_{4}\right)_{x x y}+\left(u C_{5}\right)_{x x z}+\left(u C_{6}\right)_{y y z}+\left(u C_{7}\right)_{y z z}-\left(u D_{1}\right)_{x y}- \\
-\left(u D_{2}\right)_{x z}-\left(u D_{3}\right)_{y z}+\left(u E_{1}\right)_{x}+\left(u E_{2}\right)_{y}+\left(u E_{3}\right)_{z}-\left(u D_{4}\right)_{z z}-\left(u D_{5}\right)_{y y}- \\
-\left(u D_{6}\right)_{x x}+\left\{u\left(a_{002} R\right)_{z}+u_{x}\left(a_{102} R\right)_{z}+u_{y}\left(a_{012} R\right)_{z}+u_{x y}\left(a_{112} R\right)_{z}+u_{x x}\left(a_{202} R\right)_{z}+\right. \\
\left.+u_{y y}\left(a_{022} R\right)_{z}+u_{x x y}\left(a_{212} R\right)_{z}+u_{x y y}\left(a_{122} R\right)_{z}+u_{x x y y} R_{z}\right\}_{z}+\left\{u\left(a_{202} R\right)_{x z}+u_{y}\left(a_{212} R\right)_{x z}+\right. \\
\left.+u_{y y} R_{x z}\right\}_{x z}+\left\{u\left(a_{022} R\right)_{y z}+u_{x}\left(a_{122} R\right)_{y z}+u_{x x} R_{y z}\right\}_{y z}+\left\{u R_{x y z}\right\}_{x y z}+\left\{u\left(a_{200} R\right)_{x}+\right. \\
+u_{y}\left(a_{210} R\right)_{x}+u_{y y}\left(a_{220} R\right)_{x}+u_{z}\left(a_{201} R\right)_{x}+u_{z z}\left(a_{202} R\right)_{x}+u_{y z}\left(a_{211} R\right)_{x}+u_{y y z}\left(a_{221} R\right)_{x}+ \\
\left.+u_{y z z}\left(a_{212} R\right)_{x}+u_{y y z z} R_{x}\right\}_{x}+\left\{u\left(a_{020} R\right)_{y}+u_{x}\left(a_{120} R\right)_{y}+u_{z}\left(a_{021} R\right)_{y}+u_{x z}\left(a_{121} R\right)_{y}+\right. \\
\left.+u_{x x}\left(a_{220} R\right)_{y}+u_{z z}\left(a_{022} R\right)_{y}+u_{x z z}\left(a_{122} R\right)_{y}+u_{x x z}\left(a_{221} R\right)_{y}+u_{x x z z} R_{y}\right\}_{y}+ \\
+\left\{u\left(a_{220} R\right)_{x y}+u_{z}\left(a_{221} R\right)_{x y}+u_{z z} R_{x y}\right\}_{x y},
\end{gathered}
$$

где введены обозначения:

$$
\begin{aligned}
& A_{1}=R_{z}-a_{221} R, A_{2}=R_{y}-a_{212} R, A_{3}=R_{x}-a_{122} R, \\
& B_{1}=R_{x y}-\left(a_{212} R\right)_{x}-\left(a_{122} R\right)_{y}+a_{112} R, B_{2}=R_{y z}-\left(a_{221} R\right)_{y}-\left(a_{212} R\right)_{z}+a_{211} R, \\
& B_{3}=R_{x z}-\left(a_{221} R\right)_{x}-\left(a_{122} R\right)_{z}+a_{121} R, B_{4}=R_{z z}-\left(a_{221} R\right)_{z}+a_{220} R, \\
& B_{5}=R_{y y}-\left(a_{212} R\right)_{y}+a_{202} R, B_{6}=R_{x x}-\left(a_{122} R\right)_{x}+a_{022} R, \\
& C_{1}=R_{x y z}-\left(a_{221} R\right)_{x y}-\left(a_{212} R\right)_{x z}-\left(a_{122} R\right)_{y z}+\left(a_{112} R\right)_{z}+ \\
& +\left(a_{211} R\right)_{x}+\left(a_{121} R\right)_{y}-a_{111} R, \\
& C_{2}=R_{x y y}-\left(a_{212} R\right)_{x y}-\left(a_{122} R\right)_{y y}-\left(a_{112} R\right)_{y}+\left(a_{202} R\right)_{x}-a_{102} R, \\
& C_{3}=R_{x z z}-\left(a_{221} R\right)_{x z}-\left(a_{122} R\right)_{z z}-\left(a_{121} R\right)_{z}+\left(a_{220} R\right)_{x}-a_{120} R, \\
& C_{4}=R_{y z z}-\left(a_{221} R\right)_{y z}-\left(a_{212} R\right)_{z z}+\left(a_{211} R\right)_{z}+\left(a_{220} R\right)_{y}-a_{210} R, \\
& C_{5}=R_{y y z}-\left(a_{221} R\right)_{y y}-\left(a_{212} R\right)_{y z}+\left(a_{211} R\right)_{y}+\left(a_{202} R\right)_{z}-a_{201} R, \\
& C_{6}=R_{x x z}-\left(a_{221} R\right)_{x x}-\left(a_{122} R\right)_{x z}+\left(a_{121} R\right)_{x}+\left(a_{022} R\right)_{y}-a_{021} R, \\
& C_{7}=R_{x x y}-\left(a_{212} R\right)_{x x}-\left(a_{122} R\right)_{x y}+\left(a_{112} R\right)_{x}+\left(a_{022} R\right)_{y}-a_{012} R,
\end{aligned}
$$




$$
\begin{aligned}
& D_{1}=R_{x y z z}-\left(a_{221} R\right)_{x y z}-\left(a_{212} R\right)_{x z z}-\left(a_{122} R\right)_{y z z}+\left(a_{112} R\right)_{z z}+\left(a_{211} R\right)_{x z}+\left(a_{121} R\right)_{y z}+ \\
& +\left(a_{220} R\right)_{x y}-\left(a_{111} R\right)_{z}-\left(a_{120} R\right)_{y}-\left(a_{210} R\right)_{x}+a_{110} R \text {, } \\
& D_{2}=R_{x y y z}-\left(a_{221} R\right)_{x y y}-\left(a_{212} R\right)_{x y z}-\left(a_{122} R\right)_{y y z}+\left(a_{112} R\right)_{y z}+\left(a_{211} R\right)_{x y}+\left(a_{121} R\right)_{y y}+ \\
& +\left(a_{202} R\right)_{x z}-\left(a_{111} R\right)_{y}-\left(a_{102} R\right)_{z}-\left(a_{201} R\right)_{x}+a_{101} R \text {, } \\
& D_{3}=R_{x x y z}-\left(a_{221} R\right)_{x x y}-\left(a_{212} R\right)_{x x z}-\left(a_{122} R\right)_{x y z}+\left(a_{112} R\right)_{x z}+\left(a_{211} R\right)_{x x}+\left(a_{121} R\right)_{x y}+ \\
& +\left(a_{022} R\right)_{y z}-\left(a_{111} R\right)_{x}-\left(a_{021} R\right)_{y}-\left(a_{012} R\right)_{z}+a_{011} R \text {, } \\
& D_{4}=R_{x x y y}-\left(a_{212} R\right)_{x x y}-\left(a_{122} R\right)_{x y y}+\left(a_{112} R\right)_{x y}+\left(a_{202} R\right)_{x x}+\left(a_{022} R\right)_{y y}-\left(a_{102} R\right)_{x}- \\
& -\left(a_{012} R\right)_{y}+a_{002} R \text {, } \\
& D_{5}=R_{x x z z}-\left(a_{221} R\right)_{x x z}-\left(a_{122} R\right)_{x z z}+\left(a_{121} R\right)_{x z}+\left(a_{220} R\right)_{x x}+\left(a_{022} R\right)_{z z}-\left(a_{120} R\right)_{x}- \\
& -\left(a_{012} R\right)_{z}+a_{020} R \text {, } \\
& D_{6}=R_{y y z z}-\left(a_{221} R\right)_{y y z}-\left(a_{212} R\right)_{y z z}+\left(a_{211} R\right)_{y z}+\left(a_{220} R\right)_{y y}+\left(a_{202} R\right)_{z z}-\left(a_{210} R\right)_{y}- \\
& -\left(a_{201} R\right)_{z}+a_{200} R \\
& E_{1}=R_{x y y z z}-\left(a_{221} R\right)_{x y y z}-\left(a_{212} R\right)_{x y z z}-\left(a_{122} R\right)_{y y z z}+\left(a_{112} R\right)_{y z z}+\left(a_{211} R\right)_{x y z}+ \\
& +\left(a_{121} R\right)_{y y z}+\left(a_{220} R\right)_{x y y}+\left(a_{202} R\right)_{x z z}-\left(a_{111} R\right)_{y z}-\left(a_{102} R\right)_{z z}-\left(a_{120} R\right)_{y y}- \\
& -\left(a_{210} R\right)_{x y}-\left(a_{201} R\right)_{x z}+\left(a_{110} R\right)_{y}+\left(a_{101} R\right)_{z}+\left(a_{200} R\right)_{x}-a_{100} R \text {, } \\
& E_{2}=R_{x x y z z}-\left(a_{221} R\right)_{x x y z}-\left(a_{212} R\right)_{x x z z}-\left(a_{122} R\right)_{x y z z}+\left(a_{112} R\right)_{x z z}+\left(a_{211} R\right)_{x x z}+ \\
& +\left(a_{121} R\right)_{x y z}+\left(a_{220} R\right)_{x x y}+\left(a_{022} R\right)_{y z z}-\left(a_{111} R\right)_{x z}-\left(a_{120} R\right)_{x y}-\left(a_{210} R\right)_{x x}- \\
& -\left(a_{021} R\right)_{y z}-\left(a_{012} R\right)_{z z}+\left(a_{110} R\right)_{x}+\left(a_{011} R\right)_{z}+\left(a_{020} R\right)_{y}-a_{010} R \text {, } \\
& E_{3}=R_{x x y y z}-\left(a_{221} R\right)_{x x y y}-\left(a_{212} R\right)_{x x y z}-\left(a_{122} R\right)_{x y y z}+\left(a_{112} R\right)_{x y z}+\left(a_{211} R\right)_{x x y}+ \\
& +\left(a_{121} R\right)_{x y y}+\left(a_{202} R\right)_{x x z}+\left(a_{022} R\right)_{y y z}-\left(a_{111} R\right)_{x y}-\left(a_{102} R\right)_{x z}-\left(a_{201} R\right)_{x x}- \\
& -\left(a_{021} R\right)_{y y}-\left(a_{012} R\right)_{y z}+\left(a_{101} R\right)_{x}+\left(a_{011} R\right)_{y}+\left(a_{002} R\right)_{z}-a_{001} R \text {, }
\end{aligned}
$$

Здесь $a_{\alpha \beta \gamma}$ зависят от $(x, y, z)$, а $R$ и ее производные- от $(x, y, z, \xi, \eta, \varsigma)$. Получение (6) представляет собой эвристическую задачу. Само же тождество проще всего проверить непосредственным вычислением. При этом учитываем, что $R$ по первой тройке аргументов удовлетворяет сопряженному с (1) уравнению

$$
\begin{gathered}
L^{*}(V) \equiv V_{x x y y z}-\left(a_{221} V\right)_{x x y y z}-\left(a_{211} V\right)_{x x y z z}-\left(a_{121} V\right)_{x y y z z}+\left(a_{112} V\right)_{x y z z}+ \\
+\left(a_{211} V\right)_{x x y z}+\left(a_{121} V\right)_{x y y z}+\left(a_{220} V\right)_{x x y y}+\left(a_{202} V\right)_{x x z z}+\left(a_{022} V\right)_{y y z z}- \\
-\left(a_{110} V\right)_{x y z}-\left(a_{102} V\right)_{x z z}-\left(a_{120} V\right)_{x y y}-\left(a_{210} V\right)_{x x y}-\left(a_{201} V\right)_{x x z}-\left(a_{021} V\right)_{y y z}+ \\
-\left(a_{012} V\right)_{y z z}+\left(a_{110} V\right)_{x y}+\left(a_{101} V\right)_{x z}+\left(a_{011} V\right)_{y z}+\left(a_{002} V\right)_{z z}+ \\
+\left(a_{020} V\right)_{y y}+\left(a_{200} V\right)_{x x}-\left(a_{100} V\right)_{x}- \\
-\left(a_{010} V\right)_{y}-\left(a_{001} V\right)_{z}-a_{000} V=0 .
\end{gathered}
$$

Из (5) непосредственно следует, что

$$
\begin{aligned}
& A_{1}(x, y, z, x, y, z) \equiv A_{2}(x, y, z, x, y, z) \equiv A_{3}(x, y, z, x, y, z) \equiv B_{1}(x, y, z, x, y, z) \equiv \\
\equiv & B_{2}(x, y, z, x, y, z) \equiv B_{3}(x, y, z, x, y, z) \equiv B_{4}(x, y, z, x, y, z) \equiv B_{5}(x, y, z, x, y, z) \equiv \\
\equiv & C_{1}(x, y, z, x, y, z) \equiv C_{2}(x, y, z, x, y, z) \equiv C_{3}(x, y, z, x, y, z) \equiv C_{4}(x, y, z, x, y, z) \equiv \\
\equiv & C_{5}(x, y, z, x, y, z) \equiv C_{6}(x, y, z, x, y, z) \equiv C_{7}(x, y, z, x, y, z) \equiv D_{1}(x, y, z, x, y, z) \equiv \\
\equiv & D_{2}(x, y, z, x, y, z) \equiv D_{3}(x, y, z, x, y, z) \equiv D_{4}(x, y, z, x, y, z) \equiv D_{5}(x, y, z, x, y, z) \equiv \\
\equiv & D_{6}(x, y, z, x, y, z) \equiv E_{1}(x, y, z, x, y, z) \equiv E_{2}(x, y, z, x, y, z) \equiv E_{3}(x, y, z, x, y, z) \equiv 0
\end{aligned}
$$

Положим теперь в (6) $x=\alpha, y=\beta, z=\gamma, \xi=x, \eta=y, \varsigma=z$ и вычислим от правой и левой части тройной интеграл в пределах $x_{0}<\alpha<x, y_{0}<\beta<y, z_{0}<\gamma<z$. Учитывая при этом тождества (9), граничные условия (3) и то, что $u(x, y, z)$ является решением уравнения (1), приходим к соотношению: 


$$
\begin{aligned}
& u_{x y z}=\int_{x_{0}}^{x} \int_{y_{0}}^{y} \int_{z_{0}}^{z} R(\alpha, \beta, \gamma, x, y, z) d \gamma d \beta d \alpha+\varphi_{1 y z}(y, z) R\left(x_{0}, y, z, x, y, z\right)+ \\
& +\psi_{1 x z}(x, z) R\left(x, y_{0}, z, x, y, z\right)-\varphi_{1 y z}\left(y_{0}, z\right) R\left(x_{0}, y_{0}, z, x, y, z\right)+ \\
& +\theta_{1 x y}(x, y) R\left(x, y, z_{0}, x, y, z\right)-\varphi_{1 y z}\left(y, z_{0}\right) R\left(x_{0}, y, z_{0}, x, y, z\right)- \\
& -\psi_{1 x z}\left(x, z_{0}\right) R\left(x, y_{0}, z_{0}, x, y, z\right)+\varphi_{1 y z}\left(y_{0}, z_{0}\right) R\left(x_{0}, y_{0}, z_{0}, x, y, z\right)- \\
& -A_{1}\left(x_{0}, y, z\right) \varphi_{1 y}(y, z)-A_{1}\left(x, y_{0}, z\right) \psi_{1 y}(x, z)+A_{1}\left(x_{0}, y_{0}, z\right) \varphi_{1 y}\left(y_{0}, z\right)- \\
& -A_{1}\left(x, y, z_{0}\right) \theta_{x y}(x, y)+A_{1}\left(x_{0}, y, z_{0}\right) \varphi_{1 y}\left(y, z_{0}\right)+A_{1}\left(x, y_{0}, z_{0}\right) \psi_{1 x}\left(x, z_{0}\right)- \\
& -A_{1}\left(x_{0}, y_{0}, z_{0}\right) \varphi_{1 y}\left(y_{0}, z_{0}\right)-A_{2}\left(x_{0}, y, z\right) \varphi_{1 z}(y, z)-A_{2}\left(x, y_{0}, z\right) \psi_{x z}(x, z)+ \\
& +A_{2}\left(x_{0}, y_{0}, z\right) \varphi_{1 z}\left(y_{0}, z\right)-A_{2}\left(x, y, z_{0}\right) \theta_{1 x}(x, y)+A_{2}\left(x_{0}, y, z_{0}\right) \varphi_{1 y}\left(y, z_{0}\right)+ \\
& +A_{2}\left(x, y_{0}, z_{0}\right) \theta_{1 x}\left(x, y_{0}\right)-A_{2}\left(x_{0}, y_{0}, z_{0}\right) \varphi_{1 z}\left(y_{0}, z_{0}\right)-A_{3}\left(x_{0}, y, z\right) \varphi_{y z}(y, z)- \\
& -A_{3}\left(x, y_{0}, z\right) \psi_{1 z}(x, z)+A_{3}\left(x_{0}, y_{0}, z\right) \psi_{1 z}\left(x_{0}, z\right)-A_{3}\left(x, y, z_{0}\right) \theta_{1 y}(x, y)+ \\
& +A_{3}\left(x_{0}, y, z_{0}\right) \theta_{1 y}\left(x_{0}, y\right)+A_{3}\left(x, y_{0}, z_{0}\right) \psi_{1 z}\left(x, z_{0}\right)-A_{3}\left(x_{0}, y_{0}, z_{0}\right) \psi_{1 z}\left(x_{0}, z_{0}\right)+ \\
& +B_{1}\left(x_{0}, y, z\right) \varphi_{z}(y, z)+B_{1}\left(x, y_{0}, z\right) \psi_{z}(x, z)-B_{1}\left(x_{0}, y_{0}, z\right) \varphi_{z}\left(y_{0}, z\right)+ \\
& +B_{1}\left(x, y, z_{0}\right) \theta_{1}(x, y)-B_{1}\left(x_{0}, y, z_{0}\right) \theta_{1}\left(x_{0}, y\right)-B_{1}\left(x, y_{0}, z_{0}\right) \theta_{1}\left(x, y_{0}\right)+ \\
& +B_{1}\left(x_{0}, y_{0}, z_{0}\right) \theta_{1}\left(x_{0}, y_{0}\right)+B_{2}\left(x_{0}, y, z\right) \varphi_{1}(y, z)+B_{2}\left(x, y_{0}, z\right) \psi_{1 x}(x, z)- \\
& -B_{2}\left(x_{0}, y_{0}, z\right) \varphi_{1}\left(y_{0}, z\right)+B_{2}\left(x, y, z_{0}\right) \theta(x, y)-B_{2}\left(x_{0}, y, z_{0}\right) \varphi_{1}\left(y, z_{0}\right)- \\
& -B_{2}\left(x, y_{0}, z_{0}\right) \theta_{x}\left(x, y_{0}\right)+B_{2}\left(x_{0}, y_{0}, z_{0}\right) \varphi_{1}\left(y_{0}, z_{0}\right)+B_{3}\left(x_{0}, y, z\right) \varphi_{y}(y, z)+ \\
& +B_{3}\left(x, y_{0}, z\right) \psi_{1}(x, z)-B_{3}\left(x_{0}, y_{0}, z\right) \psi_{1}\left(x_{0}, z\right)+B_{3}\left(x, y, z_{0}\right) \theta_{y}(x, y)- \\
& -B_{3}\left(x_{0}, y, z_{0}\right) \varphi_{y}\left(y, z_{0}\right)-B_{3}\left(x, y_{0}, z_{0}\right) \psi_{1}\left(x, z_{0}\right)+B_{3}\left(x_{0}, y_{0}, z_{0}\right) \psi_{1}\left(x_{0}, z_{0}\right)- \\
& -C_{1}\left(x_{0}, y, z\right) \varphi(y, z)-C_{1}\left(x, y_{0}, z\right) \psi(x, z)+C_{1}\left(x_{0}, y_{0}, z\right) \varphi\left(y_{0}, z\right)- \\
& -C_{1}\left(x, y, z_{0}\right) \theta(x, y)+C_{1}\left(x_{0}, y, z_{0}\right) \varphi\left(y, z_{0}\right)+C_{1}\left(x, y_{0}, z_{0}\right) \psi\left(x, z_{0}\right)- \\
& -C_{1}\left(x_{0}, y_{0}, z_{0}\right) \varphi\left(y_{0}, z_{0}\right)+ \\
& +\int_{x_{0}}^{x}\left\{B_{6}\left(\alpha, y_{0}, z\right) \psi_{1 z}(\alpha, z)+B_{6}\left(\alpha, y, z_{0}\right) \theta_{1 y}(\alpha, y)+B_{6}\left(\alpha, y_{0}, z_{0}\right) \psi_{1 z}\left(\alpha, z_{0}\right)-\right. \\
& -C_{6}\left(\alpha, y_{0}, z\right) \psi_{1}(\alpha, z)-C_{6}\left(\alpha, y, z_{0}\right) \theta_{y}(\alpha, y)+C_{6}\left(\alpha, y_{0}, z_{0}\right) \psi_{1}\left(\alpha, z_{0}\right)- \\
& -C_{7}\left(\alpha, y_{0}, z\right) \psi_{z}(\alpha, z)-C_{7}\left(\alpha, y, z_{0}\right) \theta_{1}(\alpha, y)+C_{7}\left(\alpha, y_{0}, z_{0}\right) \theta_{1}\left(\alpha, y_{0}\right)+ \\
& \left.+D_{3}\left(\alpha, y_{0}, z\right) \psi(\alpha, z)+D_{3}\left(\alpha, y, z_{0}\right) \theta(\alpha, y)-D_{3}\left(\alpha, y_{0}, z_{0}\right) \psi\left(\alpha, z_{0}\right)\right\} d \alpha+ \\
& +\int_{y_{0}}^{y}\left\{B_{5}\left(x, \beta, z_{0}\right) \theta_{1 x}(x, \beta)+B_{5}\left(x_{0}, \beta, z\right) \varphi_{1 z}(\beta, z)-B_{5}\left(x_{0}, \beta, z_{0}\right) \varphi_{1 z}\left(\beta, z_{0}\right)-\right. \\
& -C_{2}\left(x_{0}, \beta, z\right) \varphi_{z}(\beta, z)-C_{2}\left(x, \beta, z_{0}\right) \theta_{1}(x, \beta)+C_{2}\left(x_{0}, \beta, z_{0}\right) \theta_{1}\left(x_{0}, \beta\right)- \\
& -C_{5}\left(x_{0}, \beta, z\right) \varphi_{1}(\beta, z)-C_{5}\left(x, \beta, z_{0}\right) \theta_{x}(x, \beta)+C_{5}\left(x_{0}, \beta, z_{0}\right) \varphi_{1}\left(\beta, z_{0}\right)+ \\
& \left.+D_{2}\left(x_{0}, \beta, z\right) \varphi(\beta, z)+D_{2}\left(x, \beta, z_{0}\right) \theta(x, \beta)-D_{2}\left(x_{0}, \beta, z_{0}\right) \varphi\left(\beta, z_{0}\right)\right\} d \beta+ \\
& +\int_{z_{0}}^{z}\left\{B_{4}\left(x_{0}, y, \gamma\right) \varphi_{1 y}(y, \gamma)+B_{4}\left(x, y_{0}, \gamma\right) \psi_{1 x}(x, \gamma)-B_{4}\left(x_{0}, y_{0}, \gamma\right) \varphi_{1 y}\left(y_{0}, \gamma\right)-\right. \\
& -C_{3}\left(x_{0}, y, \gamma\right) \varphi_{y}(y, \gamma)-C_{3}\left(x, y_{0}, \gamma\right) \psi_{1}(x, \gamma)+C_{3}\left(x_{0}, y_{0}, \gamma\right) \psi_{1}\left(x_{0}, \gamma\right)- \\
& -C_{4}\left(x_{0}, y, \gamma\right) \varphi_{1}(y, \gamma)-C_{4}\left(x, y_{0}, \gamma\right) \psi_{x}(x, \gamma)+C_{4}\left(x_{0}, y_{0}, \gamma\right) \varphi_{1}\left(y_{0}, \gamma\right)+ \\
& \left.+D_{1}\left(x_{0}, y, \gamma\right) \varphi(y, \gamma)+D_{1}\left(x, y_{0}, \gamma\right) \psi(x, \gamma)-D_{1}\left(x_{0}, y_{0}, \gamma\right) \varphi\left(y_{0}, \gamma\right)\right\} d \gamma+
\end{aligned}
$$




$$
\begin{aligned}
& +\int_{x_{0}}^{x} \int_{y_{0}}^{y}\left\{D_{4}\left(\alpha, \beta, z_{0}\right) \theta_{1}(\alpha, \beta)-E_{1}\left(\alpha, \beta, z_{0}\right) \theta(\alpha, \beta)\right\} d \beta d \alpha+ \\
& +\int_{x_{0}}^{x} \int_{z_{0}}^{z}\left\{D_{5}\left(\alpha, y_{0}, \gamma\right) \psi_{1}(\alpha, \gamma)-E_{2}\left(\alpha, y_{0}, \gamma\right) \psi(\alpha, \gamma)\right\} d \gamma d \alpha+ \\
& +\int_{y_{0}}^{y} \int_{z_{0}}^{z}\left\{D_{6}\left(x_{0}, \beta, \gamma\right) \varphi_{1}(\beta, \gamma)-E_{1}\left(x_{0}, \beta, \gamma\right) \varphi(\beta, \gamma)\right\} d \gamma d \beta .
\end{aligned}
$$

Отсюда, обозначив через $H(x, y, z, x, y, z)$ правую часть (10) без первого слагаемого, приходим к решению рассматриваемой задачи (1), (3):

$$
\begin{gathered}
u(x, y, z)=\varphi(y, z)+\psi(x, z)+\theta(x, y)-\varphi\left(y_{0}, z\right)-\psi\left(x, z_{0}\right)-\theta\left(x_{0}, y\right)+\varphi\left(y_{0}, z_{0}\right)+ \\
+\int_{x_{0}}^{x} \int_{y_{0}}^{y} \int_{z_{0}}^{z} H(\alpha, \beta, \gamma, \alpha, \beta, \gamma) d \gamma d \beta d \alpha+ \\
+\int_{x_{0}}^{x} \int_{x_{0}}^{\alpha} \int_{y_{0}}^{y} \int_{y_{0}}^{\beta} \int_{z_{0}}^{z} \int_{z_{0}}^{\gamma} R\left(t_{1}, t_{2}, t_{3}, \alpha, \beta, \gamma\right) F\left(t_{1}, t_{2}, t_{3}\right) d t_{3} d \gamma d t_{2} d \beta d t_{1} d \alpha .
\end{gathered}
$$

Условия гладкости (2) на коэффициенты уравнения (1) обеспечивают принадлежность решения классу $C^{2+2+2}(D)$. Если считать функции $\varphi, \varphi_{1}, \psi, \psi_{1}, \theta, \theta_{1}$ произвольными, то можно рассматривать (10)-(11) как общее представление решения уравнения (1), подобно тому, как это делается в книге $\left[15\right.$, с.66] для уравнения со старшей производной $u_{x y}$.

2. Формулы (10)-(11) дают решение задачи (1), (3) в квадратурах, если известен явный вид $R$. Укажем некоторые случаи, полученные путем непосредственного решения (5).

Пусть семь коэффициентов уравнения (1) $a_{221}, a_{212}, a_{122}, a_{112}, a_{211}, a_{121}, a_{111}$ отличны от нуля, а все остальные тождественно равны нулю. При этом (5) имеет тот же вид, что и уравнение (4) из [11]. Будем считать, что дополнительно выполняются тождества:

$$
\begin{aligned}
& \frac{\partial a_{220}}{\partial y}+a_{220} a_{121}-a_{120} \equiv 0, \frac{\partial a_{220}}{\partial y}+a_{220} a_{211}-a_{210} \equiv 0, \\
& \frac{\partial a_{121}}{\partial y}+a_{121} a_{211}-a_{111} \equiv 0, \frac{\partial a_{120}}{\partial y}+a_{120} a_{211}-a_{110} \equiv 0
\end{aligned}
$$

Тогда из [11] следует, что функция Римана имеет вид:

$$
R(x, y, z, \xi, \eta, \varsigma)=\exp \left[\int_{\varsigma}^{z} a_{220}(\xi, \eta, \gamma) d \gamma+\int_{\xi}^{x} a_{121}(\alpha, \beta, z) d \alpha+\int_{\eta}^{y} a_{211}(x, \beta, z) d \beta\right] .
$$

Если последнее тождество (12) равно не нулю, а произведению $\lambda(x) \mu(y) v(z)$ и при этом

$$
a_{220}=A(z)+\delta x y, a_{121}=B(x)+\delta y z, a_{211}=C(y)+\delta x z,
$$

то используя формулы (18), (25) из [11], получим

$$
R(x, y, z, \xi, \eta, \varsigma)=R_{0}(x, y, z, \xi, \eta, \varsigma)_{0} F_{2}(1,1, \omega),
$$

где ${ }_{0} F_{2}$ - обобщенная гипергеометрическая функция [16, с.20], а

$$
\begin{aligned}
& R_{0} \equiv \exp \left[\int_{\xi}^{x} B(\alpha) d \alpha+\int_{\eta}^{y} C(\beta) d \beta+\int_{\varsigma}^{z} A(\gamma) d \gamma+\delta(x y z-\xi \eta \varsigma)\right], \\
& \omega=\int_{\xi}^{x} \lambda(\alpha) d \alpha \int_{\eta}^{y} \mu(\beta) d \beta \int_{\varsigma}^{z} v(\gamma) d \gamma .
\end{aligned}
$$

Аналогично можно вычислить $V$ и в других случаях, когда в (5) лишь один из коэффициентов $a_{\alpha \beta \gamma}$, имея определенную структуру, отличен от тождественного нуля.

\section{БИБЛИОГРАФИЧЕСКИЙ СПИСОК}

1. Colton D. Pseudoparabolic equations in one space variable // J. Different. Equations.1972.T.12. № 3.- P.559-565. 
2. Rundell W., Stecher M. Remarks concerning the supports of solution of pseudoparabolic equation // Proc. Amer. Math. Soc.1977. T.63.№ 1. P.77-81.

3. Rundell $W$. The construction of solutions to pseudoparabolic equation in noncilyndrical domains// J. Different. Equations. 1978. T.27. №3. P.394-404.

4. Rundell W., Stecher M. The uniqueness class for the Cauchy problem for pseudoparabolic equation // Proc. Amer. Math. Soc. 1979. T.76. № 2. P.253-257.

5. Водахова В. А. Краевая задача с нелокальным условием А.М. Нахушева для одного псевдопараболического уравнения// Дифференц. уравнения. 1982. Т.18. №2. С.280-285.

6. Шхануков M. Х. Об одном методе решения краевых задач для уравнений третьего порядка// ДАН СССР. 1982. T.265. №6. C.1327-1330.

7. Солдатов А.П., Шхануков М.Х. Краевые задачи с общим нелокальным условием А.А.Самарского для псевдопараболических уравнений высокого порядка// ДАН СССР. 1987. Т.297. №3. С.547-552.

8. Жегалов В.И., Уткина Е.А. Об одном псевдопараболическом уравнении третьего порядка// Изв. вузов. Матем. 1999. №10. С.73-76.

9. Фаге М.К. Задача Коши для уравнения Бианки// Матем. сборник. 1958. Т.45. №3. С.281-322.

10. Жегалов В.И. Трехмерный аналог задачи Гурса// Неклассические уравнения и уравнения смешанного типа. Новосибирск: МГУ. 1990. С.94-98.

11. Жегалов В.И. О трехмерной функции Римана// Сибирский матем. журнал. 1997. Т.36. №5. С.1074-1079.

12. Бондаренко Б.А. Базисные системы полиномиальных и квазиполиномиальных решений уравнений в частных производных. Ташкент:ФАН,1987. 146с.

13. Фаге М.К. Операторно-аналитические функции одной независимой переменной// Тр. Моск. матем. об-ва. 1958. T.7. C.227-268.

14. Мюнти Г. Интегральные уравнения. Л. - М.: ГТТИ, Т.1.1934. 330с.

15. Бицадзе А.В. Некоторые классы уравнений в частных производных. М.: Наука , 1981. 448 с.

16. Кампе де Ферье Ж., Кемпбелл Р., Петьо Г., Фогель Т. Функции математической физики. М.: ГИФМЛ, 1963. 102c. 